\title{
Défis et évolution de l'accompagnement offert aux professeurs de langue par le Régime d'immersion en français (RIF) de l'Université d'Ottawa
}

\author{
Laurence V. Thibault \\ Ithibau2@uottawa.ca \\ ILOB, UNIVERSITÉ D'OTTAWA \\ Marc Gobeil \\ marc.gobeil@uottawa.ca \\ RÉGIME D'IMMERSION EN FRANÇAIS, UNIVERSITÉ D' OTTAWA
}

\begin{abstract}
Résumé
Depuis 2006, le Régime d'immersion en français (RIF) offre des cours d'encadrement linguistique (CEL) associés à des cours de discipline enseignés dans cinq facultés de l'université (86 programmes en 2016-2017). Cependant, les professeurs de français langue seconde (FLS) sont très rarement formés à l'enseignement propre à ce type de cours développés sur mesure en lien avec chaque cours de discipline. Pour répondre à ce défi, le RIF a mis en place un accompagnement pédagogique dont la nature et le format ont évolué au cours des dix dernières années.

En janvier 2017, le RIF a souhaité faire un bilan du service et a consulté les professeurs sous forme d'un sondage qui visait à répondre aux questions suivantes : Dans quelle mesure l'accompagnement reçu jusqu'à présent répond-il aux besoins des professeurs ? Quels sont leurs nouveaux besoins ? Comment l'accompagnement peut-il évoluer de façon à maximiser et reconnaître les savoir-faire développés par cette communauté de pratique ? Le présent article résume les caractéristiques propres aux CEL, retrace l'évolution du service d'accompagnement aux professeurs et présente la perception de ces derniers à ce sujet pour finalement dresser un bilan et envisager de nouvelles pistes.
\end{abstract}

Mots-clés : Régime d'immersion en français (RIF), accompagnement aux professeurs de langue, cours d'encadrement linguistique (CEL)

\section{Abstract}

Since 2006, French Immersion Studies (FIS) program has provided adjunct language courses paired with content courses offered in five of the university's faculties (86 programs in total in 2016-2017). However, very 
few French as a Second Language (FSL) professors have had previous training in this particular kind of teaching, which entails designing tailormade activities that are developed in conjunction with the content course. In order to address this issue, the FIS has relied on the intervention of pedagogical advisors to provide language professors with support, for which the objectives and format have evolved over the past ten years.

In January 2017, the FIS wished to make an assessment of the service and consulted the professors by means of a survey which aimed at answering the following questions. To what extent has this pedagogical support addressed professors' needs thus far? What are the new challenges? In what direction and format should the pedagogical support evolve in order to maximize and acknowledge the know-how this community has gained so that it benefits everyone? First, this article identifies the defining features of adjunct language courses and sums up the evolution of the pedagogical support. Then, it presents an analysis of the perceptions professors have of that support. Finally, it offers conclusions and a series of new initiatives for the years to come.

Key words: French Immersion Studies (FIS), pedagogical support to language professors, adjunct language courses

\section{Introduction et contexte}

L'Université d'Ottawa offre des cours d'immersion depuis 1985 d'abord selon le modèle «encadré » puis, le modèle «associé » (Knoerr, 2010). Plusieurs études au fil des ans ont examiné les particularités de l'enseignement dans ces formats (Burger, Chrétien, Gingras, Hauptman, \& Migneron, 1984; Burger, Wesche, \& Migneron 1997; Bayliss 2009.) Dans le modèle des cours associés adopté à l'Université d'Ottawa depuis 2006, les professeurs de langue assistent aux cours de discipline avec leurs étudiants pour développer les activités du cours d'encadrement linguistique (CEL). Au tout début de ces expériences en immersion, les professeurs des CEL provenaient du corps professoral régulier et possédaient donc une solide expérience en enseignement du français langue seconde. Lors de la création du Régime d'immersion en français (RIF) en 2006, la multiplication des CEL a exigé l'embauche de plusieurs professeurs à temps partiel qui, malgré leurs expériences antérieures, ne connaissaient pas l'approche particulière préconisée dans ces cours (Buchanan, Knoerr, \& Burger, 2016; Burger, Weinberg, \& Wesche, 2013). Le RIF a mis en place un service d'encadrement pédagogique qui a été proposé à l'ensemble des professeurs, qu'ils soient à temps complet ou à temps partiel. Ce service a évolué au fil des ans pour répondre aux besoins changeants. La formation initiale a été remplacée par un accompagnement continu, autant en termes humains que matériels.

Le présent article résume d'abord les caractéristiques propres aux CEL et 
retrace l'évolution du service d'accompagnement aux professeurs. Ensuite, il présente les résultats d'un sondage mené en janvier 2017 auprès des professeurs de CEL pour comprendre l'appréciation que ces derniers ont du service reçu afin de dresser un bilan et d'envisager de nouvelles pistes.

\section{Le modèle d'immersion}

Le modèle d'immersion avec les cours associés à l'Université d'Ottawa combine un cours de discipline enseigné en français et un CEL. Les cours de discipline sont destinés à des publics francophones et leurs professeurs ne connaissent pas le contexte particulier de l'enseignement à des apprenants en L2. Cela fait en sorte que les étudiants en immersion font face à de nouvelles terminologies et à des concepts spécialisés qui ne sont pas nécessairement présentés de façon optimale pour des apprenants de L2, même s'ils sont d'un niveau avancé dans cette langue.

La Figure 1 présente le modèle de l'immersion, où un CEL est associé à un seul cours de discipline et l'inscription est réservée aux étudiants anglophones inscrits dans ce cours de discipline. Le professeur du CEL, qui assiste au cours de discipline, prépare un cours de langue pour aider ces étudiants à bien comprendre la matière du cours de discipline (cours de compréhension FLS 2581) ou à s'exprimer avec aisance sur le sujet du cours de discipline (cours d'expression FLS 3581. $)^{1}$

\section{Les caractéristiques des CEL}

Les CEL présentent des caractéristiques particulières, à la croisée des objectifs d'apprentissage d'un cours de langue seconde, des exigences d'un cours de discipline donné en français et des besoins des étudiants du RIF inscrits dans ces cours.

Les CEL présentent des caractéristiques inhérentes aux cours de FLS, telles que l'hétérogénéité et la motivation personnelle. D'une part, les étudiants, provenant de différents programmes de FLS au niveau secondaire (français de base, français enrichi ou immersion française) n'ont pas tous le même niveau de langue ${ }^{2}$. D'autre part, la motivation de chaque étudiant à s'inscrire au CEL joue un rôle dans son comportement et son effort en classe. A-t-il réellement

\footnotetext{
${ }^{1}$ Par ailleurs, deux autres CEL, d'un niveau plus avancé, ciblent l'amélioration des compétences d'expression orale (FLS 4581) et écrite (FLS 4781). Cependant, ces cours rassemblent des étudiants inscrits à divers cours de discipline (auxquels le professeur de CEL n'assiste pas) et sont offerts chacun une fois par année scolaire. Comme ils ne concernent que très peu de professeurs et que les approches ressemblent davantage à des cours typiques d'expression orale et écrite en FLS (français langue seconde), ils ne font pas l'objet d'étude dans cet article.

${ }^{2}$ Les étudiants sont classés par niveau (cours de compréhension ou d'expression)
} 


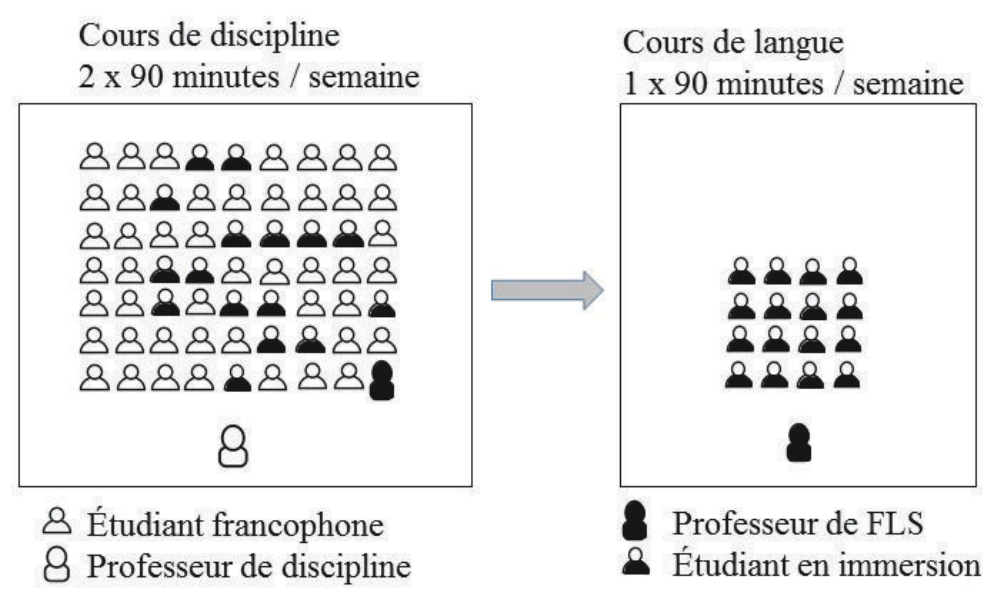

FIgURE 1

Modèle de l'immersion

besoin de l'accompagnement proposé dans le CEL, ou est-il à la recherche d'un cours où il réussira facilement? L'étudiant souhaite-t-il améliorer son français par amour pour la langue ou voit-il la langue de façon purement utilitaire?

Les CEL présentent également des caractéristiques qui leur sont propres du fait que la matière principale du cours de langue se construit à partir d'un autre cours et s'enseigne dans un cadre de temps particulièrement restreint.

D'une part, le professeur du CEL doit favoriser le développement des habiletés langagières par l'entremise d'un contenu disciplinaire sans, toutefois, enseigner ce dernier. Cette question délicate se pose aussi lors de l'évaluation des travaux des étudiants, où il faut s'assurer que ce sont les éléments linguistiques qui sont évalués et non la maîtrise de la discipline. Cependant, il n'est pas question d'approfondir certaines règles grammaticales ou de développer des connaissances culturelles générales, mais de veiller à ce que les étudiants soient outillés pour réussir le cours de discipline. Le défi consiste donc à arrimer le cours de langue au cours de discipline, à développer des objectifs langagiers et communicatifs au service des objectifs d'un autre cours (Bayliss, 2009).

en raison de leur score au test d'admission en immersion de l'Université d'Ottawa ou au Diplôme d'études en langue française (DELF) qui compilent les résultats des épreuves dans les quatre compétences langagières. Il reste cependant difficile d'assurer des groupes homogènes (Weinberg \& Hope, 2016). Par exemple, deux scores identiques peuvent représenter des combinaisons variées de niveaux de compétences en compréhension et en expression orales et écrites. 
D'autre part, le cours de discipline constitue le point de départ de toute activité dans le CEL. Le manuel ou le recueil de textes du cours de discipline, la terminologie utilisée, le cours magistral du professeur de discipline sont les sources principales du CEL. Certains cours de discipline se prêtent bien à des activités de compréhension ou d'expression, mais ce n'est pas toujours le cas. Le style d'enseignement et même les compétences linguistiques du professeur de discipline sont des éléments avec lesquels le professeur du CEL doit composer. De plus, les professeurs de discipline sont rarement habitués à avoir un autre professeur dans leur cours, (Buchanan et al., 2016) et il arrive que certains d'entre eux soient réticents à collaborer avec les professeurs de langue (Knoerr, 2010).

La gestion du temps est une caractéristique importante et problématique du CEL. Ne disposant que de quatre-vingt-dix minutes par semaine avec les étudiants, le professeur du CEL manque de temps pour travailler en profondeur les habiletés de compréhension ou d'expression. Un cours de discipline peut exiger énormément de travail au niveau de la compréhension de la terminologie, par exemple, ce qui fait en sorte que d'autres éléments ne peuvent pas être étudiés en profondeur. De plus, les activités du CEL sont souvent créées à la suite du cours de discipline. Un professeur de CEL qui a la chance d'enseigner plusieurs fois le même CEL avec le même cours de discipline aura la chance de bâtir un corpus, de réutiliser et d'affiner quelques activités, mais pour le professeur qui ne connait pas cette stabilité, le rythme soutenu de création d'activités sur mesure constitue un défi important.

La contrainte de temps devient un réel défi dans le cas des cours combinant les niveaux de compréhension et d'expression. Dans le cas de quelques CEL, les inscriptions étaient régulièrement insuffisantes dans un des deux niveaux et, par conséquent, les cours concernés étaient annulés. En 2009-2010, une expérience a été lancée où les étudiants des deux niveaux se retrouvaient dans une même salle de classe, mais avec des objectifs d'apprentissage différents. Ces « cours combinés » permettaient d'encadrer des étudiants qui, autrement, n'auraient pas bénéficié d'un CEL. Cependant, ces cours entrainaient des défis pédagogiques importants en raison de la grande variété des habiletés langagières des étudiants, du mélange des objectifs d'apprentissage et de la nécessité de faire des évaluations distinctes. Ce sujet faisait partie des questions posées aux professeurs lors du sondage en janvier 2017 et sera traité plus loin dans le présent article.

La Figure 2 présente la croissance des CEL et des cours combinés en relation avec la croissance de la population du RIF. Les étudiants sont de plus en plus nombreux dans le RIF, mais le nombre total de CEL est resté sensiblement le même d'année en année. Toutefois, c'est au niveau des cours combinés qu'une croissance remarquable est observée. 


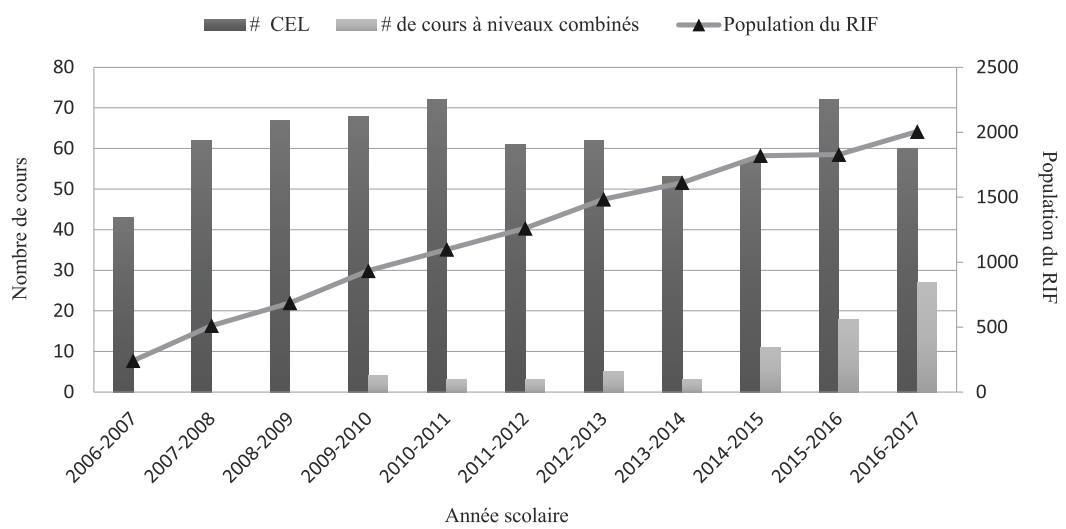

FIGURE 2

Croissance des CEL et de la population du RIF

\section{Évolution de l'accompagnement}

Comme indiqué dans l'introduction, l'Université d'Ottawa offre des cours d'immersion depuis plus de 30 ans. Les professeurs qui participaient aux premières initiatives possédaient, sans exception, plusieurs années d'expérience en enseignement au niveau universitaire, détenaient des diplômes en didactique des langues secondes, en linguistique appliquée ou en éducation et démontraient un intérêt marqué pour la discipline qui était encadrée par un CEL (Burger et al., 1997). En 2003, lorsque les CEL ont commencé à se multiplier, une embuche importante de cette méthode est apparue : les professeurs qui donnaient ces cours pour la première fois ne savaient pas quelles activités proposer aux étudiants ni comment évaluer correctement dans le cadre de ces cours (Bayliss, 2009). Lors de la création du RIF en 2006, celui-ci s'est doté d'un service d'accompagnement pédagogique dédié à former et soutenir les professeurs des CEL dans leur pratique pédagogique.

\section{Première phase}

Les premières années, le RIF a investi dans le service d'accompagnement aux professeurs en embauchant deux responsables pédagogiques recevant chacune deux dégrèvements de cours annuels car leurs tâches étaient nombreuses. Comme l'indique le premier guide à l'intention des professeurs de CEL (Weinberg \& Burger, 2006), elles organisaient et animaient des réunions d'information, des ateliers de formation, observaient les CEL et les cours de discipline, et offraient des rencontres collectives et individuelles. De plus, elles ont créé plusieurs ressources visant, d'une part, à conscientiser les professeurs 
de langues à la raison d'être des cours d'encadrement linguistique et, d'autre part, à les outiller dans leur pratique grâce à des $\mathrm{DVD}^{3}$ de formation présentant des extraits de cours de CEL et de discipline ainsi que des informations précieuses concernant les méthodes d'évaluation (Knoerr, Weinberg, \& GohardRadenkovic, 2016). Des rapports d'évaluation de programme ont confirmé le besoin critique de former et soutenir les professeurs des CEL. Ces derniers exprimaient de la confusion quant aux objectifs d'apprentissage et aux méthodes d'enseignement dont l'effet se manifestait dans l'appréciation de ces cours par les étudiants : 38\% d'entre eux étaient insatisfaits ou très insatisfaits (Ryan, Courcelles, Hope, Buchanan, \& Toews Janzen 2007; Ryan, Gobeil, Hope, Buchanan, \& Toews Janzen, 2008).

\section{Deuxième phase}

Par la suite, chacune des deux responsables pédagogiques a reçu un dégrèvement annuel pour assumer le service d'accompagnement. La deuxième phase de formation des professeurs comprenait aussi des réunions, des ateliers, ainsi que la création de documents de soutien pédagogiques et administratifs. Le système de gestion des apprentissages en ligne Campus virtuel est également devenu un outil incontournable de l'accompagnement aux professeurs. Certaines parties du guide pédagogique y ont même été mises en ligne. Cette période a surtout été marquée par le développement d'une communauté de pratique avec, comme point culminant, la création de deux recueils rassemblant des activités conçues par les professeurs des CEL désormais expérimentés : Recueil d'activités : compréhension écrite et orale d'un cours de discipline suivi en langue seconde (Dansereau \& Buchanan, 2009) et $^{4}$ 'exprimer en langue seconde dans le contexte d'un cours de discipline universitaire : recueil d'activités à l'intention des professeurs de langue en immersion postsecondaire (Dansereau, 2011). Ces recueils sont devenus des incontournables, notamment pour les nouvelles recrues qui les reçoivent automatiquement quand elles entrent en poste.

Les responsables pédagogiques ont également mené un projet sur la pertinence d'évaluer la qualité de la langue utilisée par les étudiants du cours de compréhension FLS 2581 lors de devoirs rédigés en dehors de la classe, quand ils ont accès à des outils d'autocorrection. Au cours de l'hiver 2014, les professeurs de CEL (groupe de discussion) et les étudiants des cours concernés

\footnotetext{
${ }^{3}$ Titres des DVD : L'immersion, une solution miracle ? (Burger \& Weinberg, 2006) et Testing receptive skills in immersion courses (Université d'Ottawa, s.d.).

${ }^{4}$ La deuxième édition de ce recueil, produite par les mêmes auteures en 2011, est intitulée : Comprendre un cours de discipline suivi en langue seconde : recueil d'activités à l'intention des professeurs de langue en immersion postsecondaire.
} 
(groupe de discussion et sondage) ont été consultés. Par la suite, un critère d'évaluation concernant les règles de base (telles que l'accord en genre et en nombre, l'accord entre le pronom et le verbe, l'orthographe et la conjugaison) a été ajouté au plan de cours.

De plus, c'est lors de cette période que s'est systématisée la révision des plans de cours de tous les CEL au début de chaque session universitaire afin d'assurer l'harmonisation des pratiques et le respect des spécificités des CEL, notamment en termes d'évaluation.

\section{Troisième phase}

Depuis mai 2016, seule une professeure (recevant un dégrèvement annuel) assume le rôle de responsable pédagogique. Cette réduction de l'accompagnement reflète le plus faible taux d'embauche de nouveaux professeurs. Par ailleurs, l'ensemble des professeurs des CEL a de l'expérience en immersion. Ils forment une communauté de pratique qui, si elle n'est pas tout à fait arrivée à maturité, a une base désormais solide. Aujourd'hui, l'accompagnement consiste surtout à offrir des moments de partage de pratique et des ressources en ligne grâce au Campus virtuel. Cependant, les rencontres s'avèrent difficiles à organiser en raison du grand nombre de conflits d'horaires puisqu'en plus du CEL qu'ils enseignent, les professeurs de langue doivent assister au cours de discipline associé.

Comme on peut le remarquer dans les paragraphes précédents, la raison d'être de l'accompagnement offert par le RIF aux professeurs de CEL et ses modalités d'exécution ont progressivement évolué au fil du temps.

\section{Problématique}

Après dix ans d'accompagnement offert aux professeurs de CEL, le RIF a souhaité consulter ces derniers afin de faire un bilan et d'envisager l'avenir. Le travail de réflexion et de recherche qui a été mené tout au long de l'année universitaire 2016-2017 vise à identifier, à partir des perceptions des professeurs, les forces et les faiblesses de l'accompagnement déjà offert pour consolider et renouveler celui-ci.

\section{Questions de recherche}

Les questions de recherche portent sur le passé, le présent et l'avenir de l'accompagnement offert par le RIF. Elles se formulent comme ceci : Dans quelle mesure l'accompagnement reçu jusqu'à présent répond-il aux besoins des professeurs de CEL? Quels sont leurs nouveaux besoins ? Dans quelle direction et sous quelle forme l'accompagnement peut-il évoluer de façon à maximiser et reconnaître les savoir-faire développés par cette communauté de pratique afin qu'il profite au plus grand nombre? 


\section{Méthodologie}

\section{Collecte des données}

La présente recherche suit une méthode mixte. La principale source de données pour ce travail a été un sondage envoyé aux professeurs de CEL. De plus, certaines informations complémentaires sont tirées de notre connaissance directe du terrain en tant que responsable pédagogique et directeur du RIF. Ces informations comprennent notamment des notes de réunion et des rapports internes. De plus, nous prévoyions que l'analyse des résultats du sondage influencerait directement l'accompagnement offert aux professeurs des CEL. Dans ce sens, notre démarche méthodologique s'apparente à celle d'une recherche-action.

En janvier 2017, le RIF a envoyé un sondage à tous les professeurs de langue qui ont enseigné des CEL depuis 2006. Les professeurs ont reçu l'invitation à participer au sondage par l'entremise d'un courriel envoyé le 31 janvier 2016. Un lien les dirigeait sur le site Web de SurveyMonkey, un outil qui permet de créer des sondages en ligne. Aucune date limite n'avait été fixée, mais un rappel a été envoyé après deux semaines, le 17 février. Le message a été envoyé à l'adresse courriel institutionnelle des 50 professeurs de langue qui avaient enseigné au moins un CEL depuis 2006. L'outil de sondage indique que vingt-huit personnes ont lu le courriel d'invitation (56\%), vingt ne l'ont pas ouvert (40\%) et deux adresses courriel étaient périmées (4\%). Vingt professeurs ont accédé au sondage (40\%), et finalement, dix-sept professeurs sur cinquante (34\%) ont répondu à une partie ou à la totalité du sondage.

Le sondage était composé de trente-quatre questions de types variés tels que le choix multiple, l'échelle de Likert et des questions ouvertes permettant au répondant d'inclure des commentaires. Il comprenait une première section visant à déterminer le profil du répondant, une section visant à comprendre comment ce dernier évaluait l'accompagnement et une section visant à répertorier, du point de vue du répondant, les caractéristiques et les défis propres aux cours combinés.

\section{Participants}

Sur les cinquante professeurs de CEL contactés, dix-sept ont répondu au sondage en partie ou en totalité. Huit répondants ont plus de dix ans d'expérience en enseignement des CEL (47\%), quatre ont entre sept et dix ans d'expérience $(23,5 \%)$, un seul a de quatre à six ans d'expérience $(5,9 \%)$ et quatre ont entre un et trois ans d'expérience $(23,5 \%)$. C'est donc un groupe plutôt expérimenté qui a répondu aux questions du sondage, puisque douze répondants sur dixsept $(70,5 \%)$ ont plus de sept ans d'expérience. 


\section{Méthode d'analyse}

Dans un premier temps, les réponses ont été analysées par les deux auteurs individuellement. Par la suite, quatre rencontres ont permis de combiner les analyses individuelles et de fusionner les réponses à plusieurs questions détaillées correspondant à chacune des grandes questions de recherche.

\section{Résultats et analyse}

\section{Perceptions des connaissances et des compétences}

Les professeurs de langue sont généralement formés en éducation, en didactique des langues ou en linguistique, donc ils auraient étudié les théories de Krashen, la technique de l'échafaudage et les cours encadrés, principes qui ont mené aux CEL à l'Université d'Ottawa. Toutefois, les occasions de mettre en application ces connaissances au niveau universitaire sont tout de même assez rares. De plus, l'approche des CEL est propre à notre contexte et très peu de nos professeurs de langue en avaient de bonnes connaissances avant d'enseigner un CEL.

\section{TABLEAU 1}

Connaissance de l'approche des CEL avant d'en avoir enseigné

\begin{tabular}{l|c|c|c|c|c}
\hline \hline & \multicolumn{4}{|c|}{ Années d'expérience à enseigner des CEL } & \\
\hline Connaissance de l'approche & 1 à 3 & 4 à 6 & 7 à 10 & Plus de 10 & $\mathrm{N}=16$ \\
\hline \hline Aucune connaissance & & & & & 0 \\
\hline Idée vague de l'approche & & 1 & 1 & 4 & 6 \\
$(6,25 \%)$ & $(6,25 \%)$ & $(25,00 \%)$ & $(37,50 \%)$ \\
\hline Quelques connaissances & $\begin{array}{c}2 \\
(12,50 \%)\end{array}$ & & $\begin{array}{c}1 \\
(6,25 \%)\end{array}$ & $\begin{array}{c}2 \\
(12,50 \%)\end{array}$ & $\begin{array}{c}5 \\
(31,25 \%)\end{array}$ \\
\hline Familier avec l'approche & $\begin{array}{c}2 \\
(12,50 \%)\end{array}$ & & $\begin{array}{c}1 \\
(6,25 \%)\end{array}$ & $\begin{array}{c}2 \\
(12,50 \%)\end{array}$ & $\begin{array}{c}5 \\
(31,25 \%)\end{array}$ \\
\hline \hline
\end{tabular}

Comme l'indique le Tableau 1, qui porte sur la connaissance de l'approche des CEL avant d'en avoir enseigné, seulement cinq répondants sur seize ont répondu être « familier/familière avec l'approche » $(31,25 \%)$ avant leur embauche pour un CEL, cinq répondants avaient « quelques connaissances de l'approche » $(31,25 \%)$, six avaient une «idée vague de l'approche » $(37,5 \%)$ et aucun répondant n'a déclaré être ignorant de cette approche pédagogique au moment d'enseigner son premier cours. On note que les professeurs les plus expérimentés avec les CEL sont plus nombreux à avoir déclaré qu'ils avaient une idée vague à l'aube d'enseigner leur premier CEL, ce qui peut s'expli- 
quer par le fait que l'approche préconisée n'était pas répandue il y a dix ans et que très peu de professeurs avaient déjà enseigné de cette façon. Les professeurs les moins expérimentés, c'est-à-dire les cinq personnes avec moins de six ans d'expérience avec les CEL, déclarent davantage avoir eu quelques connaissances et avoir été familiers avec l'approche. Ces professeurs ont peutêtre eu la chance d'échanger avec les collègues, de lire des articles au sujet de ces cours et de consulter les ressources qui ont été créées pour informer les nouveaux professeurs.

La Question 5 du sondage demandait aux professeurs d'évaluer leur sentiment de compétence avec l'approche des CEL à l'aube d'en enseigner. Le Tableau 2 montre que la moitié des professeurs, soit huit sur seize, se trouvait pas ou peu compétente avec les CEL avant d'en avoir enseigné et l'autre moitié se sentait compétente ou très compétente.

\section{TABLEAU 2}

Sentiment de compétence avant d'enseigner un premier CEL

\begin{tabular}{l|c|c|c|c|c}
\hline \hline & \multicolumn{4}{|c|}{ Années d'expérience à enseigner des CEL } & \\
\hline Sentiment de compétence & 1 à 3 & 4 à 6 & 7 à 10 & Plus de 10 & N = 16 \\
\hline \hline Pas compétent & & & $\begin{array}{c}1 \\
(6,25 \%)\end{array}$ & & $\begin{array}{c}1 \\
(6,25 \%)\end{array}$ \\
\hline Peu compétent & $\begin{array}{c}2 \\
(12,50 \%)\end{array}$ & $\begin{array}{c}1 \\
(6,25 \%)\end{array}$ & & $\begin{array}{c}4 \\
(25,00 \%)\end{array}$ & $\begin{array}{c}7 \\
(43,75 \%)\end{array}$ \\
\hline Compétent & $\begin{array}{c}1 \\
(6,25 \%)\end{array}$ & & 3 & $\begin{array}{c}2 \\
(12,5 \%)\end{array}$ & $\begin{array}{c}6 \\
(37,5 \%)\end{array}$ \\
\hline Très compétent & $\begin{array}{c}1 \\
(6,25 \%)\end{array}$ & & & $\begin{array}{c}1 \\
(6,25 \%)\end{array}$ & $\begin{array}{c}2 \\
(12,5 \%)\end{array}$ \\
\hline \hline
\end{tabular}

En comparant le sentiment de compétence et les années d'expérience, nous observons que quatre des sept professeurs avec plus de dix ans d'expérience se sentaient peu compétents, une donnée qui peut être expliquée par le fait que l'approche n'était pas très bien connue par les professeurs de FLS à l'Université d'Ottawa il y a dix ans. Pour ce qui est de ceux avec moins d'expérience, les données sont similaires. De fait, deux des quatre professeurs avec une à trois années d'expérience se sentaient peu compétents et les deux autres indiquaient être compétents ou très compétents.

Le Tableau 3 compare la connaissance de l'approche des CEL avant d'en avoir enseigné au sentiment de compétence. Cinq des six collègues qui avaient le moins de connaissances de l'approche s'estimaient pas ou peu compétents 
TABLEAU 3

Connaissance de l'approche et sentiment de compétence

\begin{tabular}{c|c|c|c|c|c}
\hline \hline & \multicolumn{4}{|c|}{$\begin{array}{c}\text { Sentiment de compétence } \\
\text { avant d'enseigner un CEL }\end{array}$} & \\
\hline $\begin{array}{c}\text { Connaissance } \\
\text { de l'approche }\end{array}$ & $\begin{array}{c}\text { Pas } \\
\text { compétent }\end{array}$ & $\begin{array}{c}\text { Peu } \\
\text { compétent }\end{array}$ & Compétent & $\begin{array}{c}\text { Très } \\
\text { compétent }\end{array}$ & $\mathrm{N}=15$ \\
\hline \hline $\begin{array}{c}\text { Aucune } \\
\text { connaissance }\end{array}$ & & 1 & & & 0 \\
\hline $\begin{array}{c}\text { Idée vague } \\
\text { de l'approche }\end{array}$ & $(6,7 \%)$ & $(26,7 \%)$ & $(6,7 \%)$ & & $\begin{array}{c}6 \\
(40,0 \%)\end{array}$ \\
\hline $\begin{array}{c}\text { Quelques } \\
\text { connaissances }\end{array}$ & & $\begin{array}{c}2 \\
(13,3 \%)\end{array}$ & $\begin{array}{c}2 \\
(13,3 \%)\end{array}$ & $\begin{array}{c}1 \\
(6,7 \%)\end{array}$ & $\begin{array}{c}5 \\
(33,3 \%)\end{array}$ \\
\hline $\begin{array}{c}\text { Familier avec } \\
\text { l'approche }\end{array}$ & & $\begin{array}{c}1 \\
(6,7 \%)\end{array}$ & $\begin{array}{c}2 \\
(13,3 \%)\end{array}$ & $\begin{array}{c}1 \\
(6,7 \%)\end{array}$ & $\begin{array}{c}4 \\
(26,7 \%)\end{array}$ \\
\hline \hline
\end{tabular}

$(33,4 \%)$. Les collègues avec quelques connaissances ou qui étaient familiers avec l'approche s'estimaient plus compétents.

Si certains collègues ne se sentaient pas compétents avant d'avoir enseigné un CEL, c'est tout un autre tableau à l'heure actuelle. Tous les répondants se sentent maintenant compétents $(n=11)$ ou très compétents $(n=6)$. Comment l'accompagnement reçu a-t-il pu contribuer à ce sentiment de confiance ? La prochaine section de l'article examinera de plus près l'appréciation par les professeurs des divers modes et outils d'encadrement qui leur ont été proposés au fil des ans.

\section{Appréciation de l'accompagnement déjà offert}

Dans quelle mesure l'accompagnement reçu jusqu'à présent répond-il aux besoins des professeurs? Dans l'ensemble, les ressources matérielles et les activités proposées jusqu'à présent connaissent une certaine popularité. Par ailleurs, il faut garder en tête que certains répondants ont été responsables pédagogiques, parfois dès le début du processus, et donc ont peu ou pas besoin d'accompagnement.

\section{Ressources matérielles}

La Figure 3 illustre les réponses à la question numéro 8 du sondage : « Avezvous utilisé les ressources et activités suivantes, et quelle en était votre appréciation?»

Pour chaque type de ressource, le nombre de répondants varie de dix, pour les DVD sur le RIF, à dix-sept, pour le guide des professeurs et la boîte à outils sur le Campus virtuel. Les ressources n'étant pas imposées aux professeurs, 


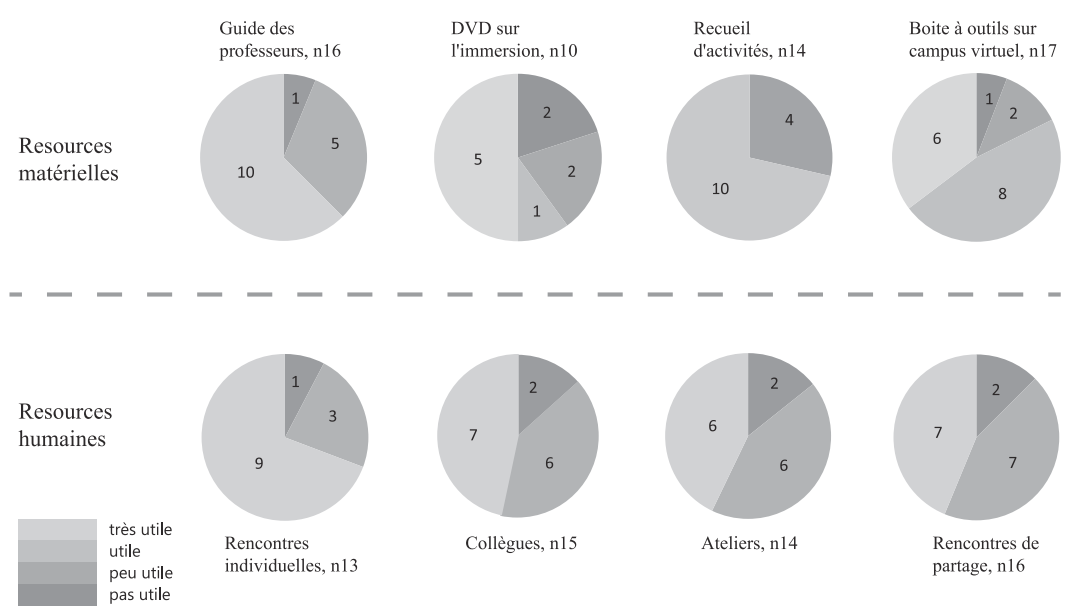

Figure 3

Appréciation des ressources et des activités

ces derniers n'ont répondu que pour les ressources qu'ils avaient consultées.

Les recueils d'activités pédagogiques sont les ressources les plus appréciées par les répondants qui ont répondu « utile » $(n=4)$, ou « très utile » $(n=$ 10 ), ce qui représente $100 \%$ de satisfaction pour ces quatorze personnes. Pour sa part, le guide des professeurs satisfait quinze répondants sur dix-sept (cinq ont répondu « utile » et dix «très utile»), soit $88,23 \%$ de satisfaction.

Il est plus difficile de conclure sur l'appréciation des autres ressources. D'une part, les DVD sur le RIF n'ont été consultés que par dix répondants et six d'entre eux ont répondu « utile » $(n=1)$ ou « très utile » $(n=5)$, soit $60 \%$ de satisfaction. D'autre part, la boîte à outils sur le Campus virtuel satisfait quatorze répondants sur dix-sept, ce qui est proche du niveau de satisfaction obtenu pour le guide des professeurs, mais avec une répartition entre «utile » $(\mathrm{n}=8)$ et « très utile » $(\mathrm{n}=6)$ différente. Il faut noter qu'il existe un chevauchement partiel entre le Campus virtuel et le guide des professeurs puisqu'une partie du guide y est reproduite. De même, le campus regroupe, en plus d'informations administratives, des informations d'ordre pédagogique dont certaines nouvelles activités créées par les professeurs et par les responsables pédagogiques. Ces dernières, généralement partagées à la suite de rencontres, ont vocation à servir de modèle de pratique. Ces croisements de contenu avec d'autres ressources populaires expliquent que la boîte à outils soit la troisième ressource la plus appréciée.

Malgré la légère disparité dans le nombre de répondants concernant chaque ressource matérielle, on peut conclure que l'ensemble des ressources offertes 
dans le cadre de l'accompagnement aux professeurs des CEL est considéré comme utile, ou très utile.

\section{Activités}

Une partie importante de l'accompagnement consiste à offrir des rencontres plus ou moins formelles aux professeurs tout au long de l'année, comme les échanges entre collègues, les rencontres individuelles entre un professeur et une responsable pédagogique, les rencontres de partage de pratiques et les ateliers de formation. Ici encore, le nombre de répondants pour chaque question varie - en l'occurrence de treize à seize. Les activités identifiées comme étant les plus satisfaisantes sont les rencontres individuelles et les rencontres de partage de pratiques, proportionnellement au nombre de répondants pour chaque item.

Les rencontres individuelles ont été utilisées par treize répondants avec un taux de satisfaction de 92,3\% (trois ont répondu « utile » et neuf, « très utile »). Cette catégorie peut englober les rencontres suite à une observation d'un CEL, une rencontre à la demande d'un professeur, une rencontre à la demande d'une responsable pédagogique pour diverses raisons, comme, par exemple un suivi concernant la révision d'un plan de cours. Par ailleurs, une autre question du sondage révèle que les observations de classe menées par les premières responsables pédagogiques ont été appréciées par huit des dix répondants concernés puisque six d'entre eux ont répondu «utile» et deux «très utile».

Pour leur part, seize répondants ont participé aux rencontres de partage de pratique avec un taux de satisfaction de $87,5 \%$ (sept ont répondu « utile » et sept «très utile»). Ces dernières années les rencontres ont eu lieu entre deux et quatre fois par session universitaire et duraient entre une heure et deux heures. Ces rencontres font généralement l'objet d'une discussion ciblée sur un élément du cours de langue pendant laquelle les collègues échangent des ressources et des exemples d'activités. Pour remédier aux conflits d'horaires, il arrive qu'une rencontre portant sur un sujet précis ait lieu deux fois. Par la suite, il est fréquent que la responsable pédagogique affiche les ressources partagées sur le Campus virtuel.

Dans la même perspective, quinze répondants précisent échanger avec leurs collègues et treize d'entre eux considèrent ces échanges utiles $(n=6)$ ou très utiles $(n=7)$ (soit $86,6 \%$ de satisfaction). Qu'il s'agisse d'échanges pendant les rencontres, à la suite ou indépendamment de celles-ci, les répondants reconnaissent leurs pairs comme des personnes ressources, notamment du fait que certains professeurs ont tendance à se spécialiser dans les CEL. Il faut également noter que parmi ces pairs, cinq différentes professeures de l'ILOB ont occupé le poste de responsable pédagogique depuis 2006 et quatre d'entre elles enseignent toujours. 
Concernant les ateliers, sur quatorze répondants, douze les ont appréciés : «utile » $(n=6)$, «très utile » $(n=6)$, et deux les ont trouvés peu utiles. Entre 2006 et 2017, ces ateliers ont traité des aspects très variés des CEL : l'évaluation, la prise de notes pendant les cours de discipline, l'utilisation de correcticiels.

L'analyse des résultats révèle donc que les répondants apprécient la plupart des ressources matérielles et les échanges en face à face et en groupe.

\section{Nouvelles habiletés et connaissances}

En plus de l'appréciation générale des ressources humaines et matérielles, nous avons demandé aux répondants si l'accompagnement leur avait apporté de nouvelles habiletés ou connaissances. Douze répondants ont complété cette partie.

D'une part, six de ces répondants (50\%) affirment que l'accompagnement leur a apporté de nouvelles habiletés concernant le développement d'activités d'apprentissage et d'évaluation mieux adaptées aux CEL, notamment grâce à une meilleure utilisation des sites et des logiciels présentés lors de rencontres et d'ateliers. D'autre part, sept des douze répondants $(58,3 \%)$ ont affirmé que l'accompagnement leur avait apporté de nouvelles connaissances au sujet des outils disponibles et une meilleure compréhension des CEL et de la méthodologie qu'on y enseigne, comme par exemple, la prise de notes, le résumé ou la fiche de lecture.

Pour résumer le taux de satisfaction général concernant l'ensemble des services d'accompagnement, nous avons posé la question suivante : «Dans quelle mesure l'accompagnement reçu jusqu'à présent répond-il à vos besoins? » Comme on peut le voir dans la Figure 4, sur quatorze répondants, trois ont répondu « très satisfaisant », six « satisfaisant », un «peu satisfaisant», et quatre ont répondu « sans objet». Il faut garder en tête que certains répondants ont été responsables pédagogiques, parfois depuis le début. Ils ont donc peu ou pas besoin d'accompagnement.

\section{Nouveaux besoins}

Un défi autrefois marginal a récemment pris de l'ampleur. La Figure 1 en début de texte affiche le nombre de CEL au fil des ans, y compris l'arrivée et l'augmentation en nombre de ce qui a été nommé les «cours combinés». La multiplication de ces cours regroupant le niveau « compréhension » (FLS 2581) et le niveau «expression» (FLS 3581) accentue le sentiment de manque de temps déjà identifié. Cette situation oblige les professeurs à répondre à deux séries d'objectifs dans le même temps d'enseignement et à évaluer des compétences différentes lors des travaux pratiques, quiz, devoirs et examens. Pour traiter ce problème spécifique, une série de rencontres a été organisée au cours de l'automne 2016 afin de partager entre collègues les manières de surmonter 


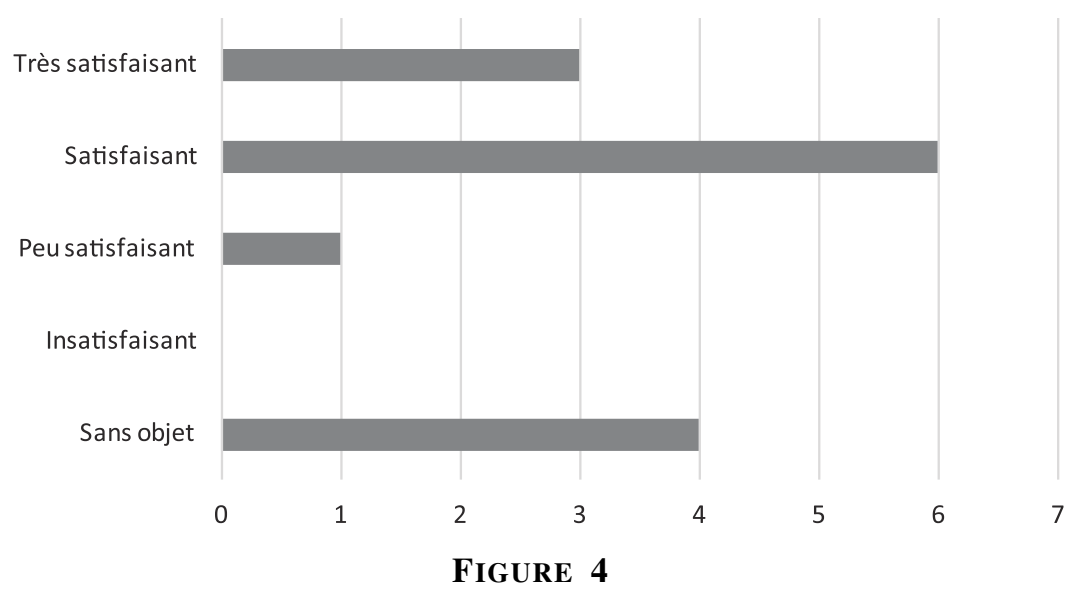

Taux de satisfaction par rapport à l'accompagnement

ce défi qui tend à être plus fréquent que par le passé. Parmi les participants se trouvaient des professeurs ayant déjà enseigné un ou plusieurs cours combinés ainsi que des professeurs enseignant ce genre de cours pour la première fois et même des professeurs qui n'étaient pas directement concernés, mais souhaitaient se former.

Dans le contexte du présent sondage, onze répondants ont indiqué avoir déjà enseigné un cours combiné et dix d'entre eux ont participé aux rencontres de partage de pratiques portant sur les cours combinés. Neuf répondants ont précisé leur niveau de satisfaction à propos de ces rencontres. Huit d'entre eux les ont trouvées utiles $(n=4)$ ou très utiles $(n=4)$, et un répondant les a trouvées peu utiles. Un commentaire a souligné l'importance de rendre les rencontres plus systématiques et un autre appelait à plus de rétroaction sur les difficultés rencontrées en classe. Le partage de pratiques s'est prolongé au-delà des rencontres grâce à la mise en ligne sur le Campus virtuel d'activités créées par les collègues dans le but d'aider les professeurs qui devront enseigner des cours combinés à l'avenir. Des documents ont également été développés pour guider les professeurs dans la création et l'évaluation des activités et des examens pour cours combinés.

Nous avons demandé aux répondants s'ils pouvaient identifier des aspects positifs dans l'approche de niveaux combinés. Ce fut le cas de cinq répondants sur dix. Toutefois ces aspects sont principalement d'ordre administratif et logistique. L'exemple le plus souvent cité est le fait de pouvoir maintenir un cours qui rassemble deux effectifs de cours trop faibles pour être maintenus comme deux cours séparés. Par ailleurs, huit répondants sur dix ont déclaré avoir «trouvé une façon de tirer profit du fait d'avoir des étudiants de niveaux 
différents dans la même classe » comme, par exemple, le jumelage entre étudiants de chaque niveau ou des tâches complémentaires comprenant un travail de présentation orale ou écrite pour les étudiants du niveau FLS 3581 (expression) face aux étudiants du niveau FLS 2581 (compréhension). C'est effectivement ce genre d'arrangements que les professeurs ont mentionné lors des rencontres de partage de pratiques au cours de l'automne 2016.

Toutefois, huit répondants sur neuf ont également déclaré identifier « des aspects négatifs » comme la trop grande diversité d'objectifs, le temps trop long de double préparation, la difficulté à développer des activités de classe pour deux niveaux et même la cohabitation entre les étudiants de niveaux différents.

Dans l'ensemble, les répondants concernés ne pensent pas que cette formule soit favorable à l'apprentissage, comme en témoigne le Tableau 4.

\section{TABLEAU 4}

Perception de l'apprentissage des étudiants dans les CEL combinés

\begin{tabular}{lcc}
\hline \hline Choix de réponse & \multicolumn{2}{l}{$\mathrm{N}=9$} \\
\hline \hline Ils apprennent beaucoup mieux & 0 & $(0,0 \%)$ \\
\hline Ils apprennent mieux & 1 & $(11,1 \%)$ \\
\hline C'est pareil & 2 & $(22,2 \%)$ \\
\hline Ils apprennent moins bien & 5 & $(55,6 \%)$ \\
\hline Ils apprennent beaucoup moins bien & 1 & $(11,1 \%)$ \\
\hline \hline
\end{tabular}

\section{Avenir de l'accompagnement}

Notre troisième volet de recherche vise à explorer des pistes d'avenir pour l'accompagnement d'une communauté de pratique désormais expérimentée. Nous avons cherché à comprendre sous quelle forme l'accompagnement peut évoluer, notamment de façon à maximiser et reconnaître les savoir-faire développés par les membres de cette communauté. D'après les réponses et les commentaires, l'ensemble de l'accompagnement déjà offert devrait rester en place et être enrichi de nouvelles modalités.

\section{Formation initiale}

Concernant les améliorations «pour former initialement un nouveau professeur de CEL » onze répondants ont fourni des commentaires dont plusieurs confirment l'importance de continuer ou de systématiser à nouveau des initiatives mises en place en 2006, soit une combinaison de formation préalable 
obligatoire et d'ateliers, de suivi individuel et d'échanges de pratiques, comme en témoignent les extraits suivants ${ }^{5}$ :

«Au minimum une séance de formation sur les objectifs, les écueils à éviter, et des exemples concrets d'activités pédagogiques. »

«Demander au nouveau professeur d'assister à une formation obligatoire (d'une journée) avant de commencer à enseigner, accompagner de façon individuelle le professeur pendant sa première session à plusieurs reprises pendant le trimestre. »

«Reprendre tous les éléments qui étaient disponibles il y a dix ans dont les visionnements du DVD, une formation obligatoire suivie d'une observation en salle de classe. »

«Plus d'échanges avec les collègues. »

«Des lectures d'articles publiés par les experts sur le sujet pour une formation à l'approche/ la philosophie ; des ateliers pour une formation pratique, une observation de classe pour une rétroaction des recommandations à mettre en œuvre ; une observation de suivi pour vérifier la mise en place des recommandations. »

Une autre série de commentaires mentionne des formules innovantes comme le parrainage, la simulation et l'accès à des échanges en ligne :

« Suivre une séance-info avec un professeur d'encadrement avec beaucoup d'expérience, ou encore participer à un cours-simulation de cours de discipline et FLS. »

«Les cours combinés demanderaient plus de formation. »

«Une plateforme interactive, un forum en ligne ou un groupe Facebook fermé. »

On note donc, d'une part, un attachement aux mesures déjà offertes ainsi qu'une tendance à la collaboration qui permet d'envisager les collègues comme des conseillers valables. Ces commentaires proposent une piste possible pour l'avenir de l'accompagnement des nouveaux professeurs de CEL par leurs pairs.

\section{Ressources et activités}

Concernant de nouvelles ressources possibles, nous avons proposé des exemples et offert l'occasion de faire des propositions (Tableau 5). Les répondants pouvaient cocher plusieurs réponses.

Un répondant a proposé des «banques de documents audio/vidéo et de sites Internet pour les différentes disciplines. » Ce commentaire évoque la difficulté qu'ont les professeurs à trouver et à renouveler des documents authentiques appropriés au développement d'activités d'apprentissage de la langue.

\footnotetext{
${ }^{5}$ Les commentaires reçus ont été classés par catégories d'idées. Seul un commentaire par catégorie est cité.
} 
TABLEAU 5

Nouvelles ressources

\begin{tabular}{llc}
\hline \hline Choix de réponse & \multicolumn{2}{c}{$\mathrm{N}=14$} \\
\hline \hline $\begin{array}{l}\text { Site web de partage (banque d'activités pédago- } \\
\text { giques, coin des experts, forum) }\end{array}$ & 11 & $(78,6 \%)$ \\
\hline Banque lexicale par cours de discipline & 10 & $(71,4 \%)$ \\
\hline $\begin{array}{l}\text { Description des connaissances de base pour les cours } \\
\text { de discipline }\end{array}$ & 7 & $(50,0 \%)$ \\
\hline Autre (veuillez préciser) & 1 & $(7,1 \%)$ \\
\hline \hline
\end{tabular}

Cette difficulté ressort particulièrement dans les CEL qui accompagnent des cours techniques souvent perçus comme étant plus difficiles à accompagner que d'autres. Par exemple, des cours comme la biologie cellulaire, la gestion financière ou la macroéconomie, pour n'en nommer que quelques-uns, posent un défi aux professeurs qui ont fait des études supérieures en didactique ou en linguistique, car ils ne possèdent que très rarement les connaissances de base pour ces cours. Contrairement aux cours qui n'exigent pas de préalables et qui sont accessibles à tous, comme l'anthropologie, la communication ou l'histoire du Canada, les cours techniques demandent certaines connaissances en sciences ou en mathématiques. Les professeurs des CEL doivent alors développer des activités en lien avec ces contenus qui peuvent, à l'occasion, leur poser un défi au niveau de la compréhension de la matière et du repérage de ressources audio et vidéo pertinentes.

Concernant les activités proposées (Tableau 6), ce sont les rencontres de partage de pratiques qui sont le plus demandées, suivies des ateliers interactifs, puis des réunions d'information et des observations en classe.

TABLEAU 6

Activités

\begin{tabular}{lrc}
\hline \hline Choix de réponse & \multicolumn{2}{c}{$\mathrm{N}=16$} \\
\hline \hline Ateliers interactifs & 10 & $(62,5 \%)$ \\
\hline Réunions d'information & 9 & $(56,3 \%)$ \\
\hline Rencontres de partage de pratiques & 12 & $(75,0 \%)$ \\
\hline Observation en classe & 6 & $(37,5 \%)$ \\
\hline \hline
\end{tabular}




\section{Personnes ressources}

Deux questions supplémentaires nous ont permis d'identifier une ressource interne à la communauté des professeurs de CEL. Comme nous l'avons vu plus haut, les répondants ont le sentiment d'être compétents dans leur enseignement d'un CEL. Sept répondants sur quatorze se sentent prêts à être « une personne ressource pour un cours de discipline ». Sept répondants sur treize acceptent d'être « une personne ressource pour un mode d'enseignement» (Tableau 7).

\section{TABLEAU 7}

Personnes ressource pour un mode d'enseignement

\begin{tabular}{lcc}
\hline \hline Choix de réponse & \multicolumn{2}{c}{$\mathrm{N}=13$} \\
\hline \hline Non & 6 & $(46,1 \%)$ \\
\hline Oui - présentiel & 5 & $(38,5 \%)$ \\
\hline Oui —à distance & 2 & $(15,4 \%)$ \\
\hline Oui — apprentissage par l'engagement communautaire & 2 & $(15,4 \%)$ \\
\hline Oui - parrainage & 3 & $(23,1 \%)$ \\
\hline Oui_-médias sociaux & 1 & $(7,7 \%)$ \\
\hline \hline
\end{tabular}

Dans le Tableau 7, l'intitulé «parrainage » décrit le parrainage d'étudiants francophones du cours de discipline auprès d'étudiants du CEL. Cette formule doit être encadrée par le professeur du cours de langue en accord avec le professeur du cours de discipline. Aussi bien pour les ressources que pour les activités, c'est la tendance au partage qui est ressortie avec les choix du site collaboratif, des rencontres de partage de pratiques et le fait de se projeter comme personne ressource pour ses collègues, y compris ceux nouvellement embauchés.

\section{Conclusion}

Dans cet article, nous avons dressé un bilan du service d'accompagnement pédagogique offert par le RIF depuis dix ans aux professeurs de CEL, notamment grâce à un sondage mené en 2017. Malgré le petit nombre de répondants $(\mathrm{n}=$ 17), nous avons pu tirer plusieurs conclusions valides du fait que notre expérience du terrain en tant que responsable pédagogique et directeur du RIF nous a permis de mettre les résultats en contexte :

- Premièrement, le sondage a révélé que les répondants estiment faire preuve de meilleures connaissances et compétences que lorsqu'ils ont commencé à enseigner des CEL. De plus, ils apprécient généralement 
les ressources et les activités offertes jusqu'à présent par le service d'accompagnement.

- Deuxièmement, les répondants considèrent que les cours combinés imposent des contraintes excessives au niveau de l'enseignement sans pour autant offrir de bonnes conditions d'apprentissage aux étudiants. Dès la rentrée de septembre 2017, le RIF a considérablement réduit le nombre de cours combinés, mais seulement dans les disciplines où les étudiants peuvent choisir parmi plusieurs CEL. Dans les disciplines où les étudiants sont moins nombreux et où les CEL à un seul niveau peuvent ne pas être viables, les cours combinés sont maintenus en attendant de trouver une meilleure solution. À long terme, il est souhaité que l'offre des cours combinés soit éliminée. Dans ce but, il faudra analyser les tendances d'inscriptions, les niveaux de langue des étudiants, les préalables aux cours et les exigences du RIF.

- Troisièmement, concernant l'avenir du service d'accompagnement, les réponses font ressortir une préférence pour le mode collaboratif, aussi bien pour compléter la formation initiale déjà en place que pour parfaire la pratique d'enseignement. Cette collaboration permettrait également de faciliter l'accès à des ressources d'ordre didactique et en lien avec les sujets des cours de discipline.

Le travail de réflexion mené en 2016-2017 pendant les rencontres avec les professeurs et lors de l'analyse du sondage a permis de consolider et de redéfinir les grands axes de l'accompagnement offert aux professeurs des CEL et d'envisager de nouvelles pistes dont certaines sont en développement depuis la rentrée de septembre 2017. La quatrième phase d'accompagnement pédagogique se définit comme suit.

D'une part, l'accompagnement assurera la pérennité d'actions qui ont fait leurs preuves au fil des ans. Il continuera d'offrir une formation initiale aux nouveaux professeurs, avec, comme modalité innovante, un travail en tandem avec un professeur expérimenté, au besoin. Les réunions d'information de prérentrée resteront également un élément stable de l'accompagnement, ainsi que la révision des plans de cours.

D'autre part, la responsable pédagogique agira comme modératrice d'une communauté de pratique, aussi bien pour l'organisation des activités que pour le développement des ressources en ligne, rassemblées dans un site collaboratif auquel les professeurs pourront contribuer (Figure 5).

Dès l'été 2017, des professeures de CEL ont été identifiés comme personnes ressources. Ainsi, une professeure de CEL a animé un atelier sur les stratégies encourageant les étudiants des CEL à poursuivre leur apprentissage 


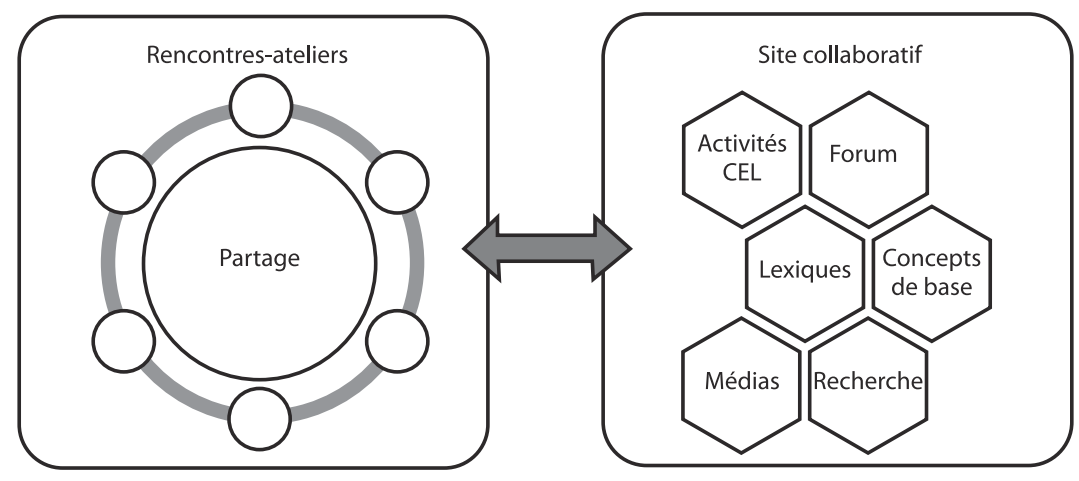

FIGURE 5

Communauté de pratique

en dehors de la salle de cours. Le sujet de l'atelier était très pertinent pour les CEL étant donné le temps restreint d'enseignement en classe d'une heure et trente minutes par semaine. Deux autres professeurs ont accepté d'intervenir pendant la session d'hiver 2018. Ces ateliers ont lieu en alternance avec des rencontres de partage de pratiques deux à quatre fois par session.

Le service d'accompagnement vise également à enrichir le fonds de ressources accessibles sur le Campus virtuel. Le site offrira des références sur la recherche en immersion, des liens pertinents vers des sites spécialisés offrant des documents utiles au développement d'activités de langue, des conseils aussi bien au sujet de l'enseignement de la langue (lexiques, activités CEL) qu'au sujet du contenu des cours de discipline (concepts de base, notamment pour les cours dits « difficiles»). Cette pratique sera en partie facilitée par la mise en place de plusieurs fils de discussion thématiques dans un forum où les professeurs pourront échanger directement sur le Campus virtuel des cours d'immersion qui servira de site collaboratif. Celui-ci pourra également être utile pour se mettre à jour en cas d'absence étant donné le grand nombre de conflits d'horaires.

En ce qui concerne les professeurs de discipline, le RIF poursuivra ses démarches pour les sensibiliser aux défis des étudiants de langue seconde, au rôle joué par le professeur de CEL, ainsi qu'à leur propre rôle dans l'encadrement offert aux étudiants et aux professeurs de langue. En plus de la lettre d'informations et de conseils envoyée en début de chaque session, le directeur du RIF a rencontré les conseils des différentes facultés au cours de l'automne 2017 et prévoit d'informer les professeurs lors des assemblées départementales en 2018. Ces derniers seront aussi invités à contribuer, sur une base volontaire, à la création de lexiques propres à leur discipline ou à leur cours, transmettant 
des informations qui seront utiles à la fois aux étudiants et aux professeurs de langue. De plus, les professeurs de discipline seront invités à partager avec le RIF des sources d'information accessibles et pertinentes (en français) que les professeurs de langue pourront utiliser dans leurs CEL.

Le modèle collaboratif qui se met progressivement en place depuis l'automne 2017 permettra, à terme, de remédier à certains des défis occasionnés par la réduction des ressources humaines affectées à l'accompagnement entre 2006 et 2016. Cette réduction affecte la fréquence des rencontres de groupe et ralentit l'enrichissement du Campus virtuel dans une phase où celui-ci est appelé à devenir un élément central de communication. À la lumière des commentaires recueillis lors du sondage de 2017, le nombre de tâches à réaliser amène le RIF à envisager une évolution en deux étapes. Dans un premier temps, il faudra augmenter le nombre de responsables pédagogiques pour développer les sections interactives du site. Dans un deuxième temps, les professeurs pourront prendre le relais en partageant des ressources et en ayant des échanges grâce au forum. Une nouvelle évaluation des ressources sera alors nécessaire pour mesurer l'efficacité du site et la satisfaction de ses utilisateurs.

\section{Références}

Bayliss, D. (2009). The implementation of a program of immersion courses : What we have learned so far. Dans L. Yu (dir.), Bilingual instruction in China : A global perspective - Papers from the Symposium on Canadian Immersion Education and Bilingual Instruction at the Tertiary Level in China. Beijing, Foreign Language Teaching and Research Press, pp. 22-40.

Buchanan, C.É., Knoerr, H., et Burger, S. (2016). La formation des divers acteurs de l'immersion. Dans H. Knoerr, A. Weinberg, et A. Gohard-Radenkovic (dir.), L'immersion française à l'université : Politiques et pédagogies. Ottawa, Les Presses de l'Université d'Ottawa, pp. 313-345.

Burger, S., Chrétien, M., Gingras, M., Hauptman, P., et Migneron, M. (1984). Le rôle du professeur de langue dans un cours de matière académique en langue seconde. La Revue canadienne des langues vivantes, 41, pp. 397-402.

Burger, S. Wesche, M., et Migneron, M. (1997). Late, late immersion : Disciplinebased second language teaching at the University of Ottawa. Dans R.K. Johnson et M. Swain (dir.), Immersion education : International perspectives. Cambridge, Cleveland University Press, pp. 65-84.

Burger, S., et Weinberg, A. (2006). L'immersion, une solution miracle? DVD sur l'immersion à l'université. Ottawa, Université d'Ottawa, Institut des langues secondes.

Burger, S., Weinberg, A., et Wesche, M. (2013). Immersion studies at the University of Ottawa : From the 1980s to the present. Cahiers de l'ILOB, 6, pp. 21-43.

Dansereau, M.-C. (dir.) (2011). S'exprimer en langue seconde dans le contexte d'un cours de discipline universitaire : recueil d'activités à l'intention des professeurs 
de langue en immersion postsecondaire. $2^{\mathrm{e}}$ éd. Ottawa, Institut des langues officielles et du bilinguisme, Université d'Ottawa.

Dansereau, M.-C., et Buchanan, C.É. (dir.) (2009). Recueil d'activités : compréhension écrite et orale d'un cours de discipline suivi en langue seconde. Ottawa, Institut des langues officielles et du bilinguisme, Université d'Ottawa.

Dansereau, M.-C., et Buchanan, C.É. (dir.) (2011). Comprendre un cours de discipline suivi en langue seconde : recueil d'activités à l'intention des professeurs de langue en immersion postsecondaire. $2^{\mathrm{e}}$ éd. Ottawa, Institut des langues officielles et du bilinguisme, Université d'Ottawa.

Knoerr, H. (2010). L'immersion au niveau universitaire : nouveaux modèles, nouveaux défis, pratiques et stratégies. Cahiers de l'ILOB, 1, pp. 89-110.

Knoerr, H., Weinberg, A., et Gohard-Radenkovic, A. (dir.). (2016). L'immersion française à l'université : politiques et pédagogies. Ottawa, Presses de l'Université d'Ottawa.

Ryan, W., Courcelles, Hope, A., Buchanan, C.É., \& Toews Janzen, M. (2007). Evaluation of the French Immersion Studies Academic Stream. Document interne, Institut des langues officielles et du bilinguisme, Université d'Ottawa.

Ryan, W., Gobeil, M., P., Hope, A., Buchanan, C.É., \& Toews Janzen, M. (2008). Evaluation of the French Immersion Studies Academic Stream : Year 2. Document interne, Institut des langues officielles et du bilinguisme, Université d'Ottawa.

Université d'Ottawa. (s.d.). «DVD», Testing receptive skills in immersion courses. Institut de langues officielles et du bilinguisme, Université d'Ottawa.

Weinberg, A., et Burger, S. (2006). Guide à l'intention des professeurs de langues : cours d'immersion. Ottawa, Institut des langues secondes, Université d'Ottawa.

Weinberg A., et Hope, A. (2016). Structures et modalités d'évaluation au sein du Régime d'immersion en français. Dans H. Knoerr, A. Weinberg, et A. GohardRadenkovic (dir.), L'immersion française à l'université : Politiques et pédagogies. Ottawa, Les Presses de l'Université d'Ottawa, pp. 285-311. 